\title{
ALGUNAS NOTAS SOBRE LAS REVISTAS FRANCESAS DE DIVULGACIÓN HISTÓRICA. EL CASO DE L'HISTOIRE
}

\section{Some notes about the French historical magazines. The case of L'Histoire}

\author{
Jean-Louis GuEREÑA \\ ICD. Université de Tours \\ Correo-e: jean-louis.guerena@univ-tours.fr \\ Recibido: II de enero de 202I \\ Envío a informantes: 16 de enero de 202I \\ Aceptación definitiva: 6 de marzo de 202I
}

Resumen: Dentro del «mercado de la historia» en Francia, presentamos primero algunas de las diferencias más notables entre las revistas académicas y las revistas de divulgación histórica para centrarnos a continuación en la revista L'Histoire, en la cual podemos apuntar características de unas y de otras revistas, lo cual la convierte realmente en un caso aparte. Pero la revista ha perdido en parte su estatuto primitivo de revista de vulgarización de alto nivel para adoptar una postura normativa del periodismo intelectual observable también en otras revistas.

Palabras Clave: divulgación; Francia; historiografía; revistas.

Aвstract: Within the «market of history» in France, we first present some of the most notable differences between academic journals and historical popularization journals to then focus on L'Histoire magazine in which we can point out characteristics of one and the other magazines, which really makes it a case of its own. But the magazine has partly lost its original status as a high-level popularizing magazine to adopt a normative stance of intellectual journalism observable in other magazines as well.

KEY wORDS: popularization; France; historiography; magazines. 
Para Fernando Sánchez Marcos (1943-2020), hombre de consenso, siempre interesado por la historiografía y la cultura histórica, esta modesta aportación a un tema que expuse muchas veces en sus clases.

\section{Introducción}

$\mathrm{E}$ L 9 DE MAYO DE 1978, veía la luz en los kioscos franceses el primer número de una nueva revista mensual de divulgación histórica, titulada sencillamente L'Histoire [La Historia], siempre presente y activa tras una trayectoria de más de cuarenta años y un total de 478 números «normales» publicados a lo largo de este periodo, más varios números «especiales» y otras modalidades de difusión a las cuales nos referiremos (destacando la reciente salida de L'Histoire Juniors)'.

Se trata, sin lugar a dudas, de una revista (magazine en francés) de calidad -habría que evocar así mismo la página web de la revista- ${ }^{2}$, que ocupa un lugar intermedio entre las revistas académicas de la especialidad -escritas por especialistas para especialistas- y las revistas de divulgación o de vulgarización, dirigidas a un gran público interesado por la historia y, por lo general, redactadas por periodistas, más o menos bien informados.

Esta característica específica de la revista («Le magazine de référence des passionnés d'histoire», según lo publica la revista misma [ «La revista de referencia de los apasionados por la historia»] y que podríamos sintetizar por pasión y seriedad), que explica sin duda su éxito (con una difusión considerable que ha oscilado entre los 79.000 y los 52.000 ejemplares mensuales), merece algún comentario, por rápido que sea.

Dentro de lo que podemos calificar de «mercado de la historia» (que comprende los libros, por supuesto, las revistas, las películas, pero también los espectáculos históricos...), se trata de responder de una manera o de otra a la «demanda de historia» expresada de varias maneras por parte de un amplio público, aficionado a la historia y a su «escritura», lo que conduce a plantearse (y a intentar contestarlas) las preguntas aparentemente sencillas, pero que no lo son, "para quién/quiénes se escribe», "para quién/quiénes se publica», teniendo en cuenta, además, la masa de informaciones y de interpretaciones históricas, más o menos controladas, que circulan por Internet.

Dejamos aquí de lado los espectáculos de reconstituciones históricas -como el que se celebra en la localidad del «Puy du Fou», en la Vendée (Vandea), al oeste del país-, y que es uno de los más concurridos (pero los hay en torno a Napoleón I o al desembarco de las tropas angloamericanas en Normandía en junio de 1944), las cadenas de televisión especializadas en historia o los cómics históricos para ceñirnos a las revistas de historia, que representan, pues, solo una parte de la oferta de contenido histórico de la que pueden disfrutar los aficionados a la historia, fuera, pues, de la que se dirige, principal y casi únicamente, a los profesionales de la historia.

Lebrun, F. (1998, abril). Naissance de L'Histoire. L'Histoire, Paris, 220, 7; Winock, Michel (2008, mayo). Je me souviens... c'était en 1978. Ibid., 33I, 5-6. Ver JAMET, M. (1983). La Presse périodique en France (p. 89). Paris: Armand Colin, y Senelier, A. (2008). Histoire (L'). En J. Julliard y M. Winock (eds.), Dictionnaire des intellectuels français : les personnes, les lieux, les moments (pp. 710-7II). Paris: Le Seuil.

https://www.lhistoire.fr/. 
Dentro del «mercado de la historia», presentamos primero algunas de las diferencias más notables entre las revistas académicas y las revistas de divulgación histórica para centrarnos a continuación en la revista L'Histoire, en la cual podemos apuntar características de unas y de otras revistas, lo cual la convierte realmente en un caso aparte. Pero la revista ha perdido en parte su estatuto primitivo de revista de vulgarización de alto nivel para adoptar una postura normativa del periodismo intelectual observable también en otras revistas ${ }^{3}$.

\section{Revistas académicas y revistas de divulgación}

En general, existe un mundo y, por lo tanto, una barrera prácticamente infranqueable entre, por una parte, las revistas académicas de historia y, por otra, las revistas de divulgación histórica. Todos los que trabajan en el mundo universitario conocen las revistas universitarias, difícilmente legibles, a decir verdad, por quienes no participan de una manera u otra en ese mundo (por la acumulación de notas y de referencias eruditas); las revistas pensadas y escritas por y para un público universitario especializado, lo que implica, por ejemplo, un estricto protocolo de revisión de artículos «por pares». Por citar tan solo unas cuantas: la Revue Historique, los Annales, la Revue d'Histoire Moderne et Contemporaine...

La diferencia entre unas y otras es de contenido, por supuesto, pero también de presentación y de modalidad de difusión. Una simple ojeada a un kiosco francés de prensa permite observar la cantidad de títulos de revistas que tienen que ver, de una manera u otra, con la historia y, por lo tanto, con la demanda de historia. Algunos de esos títulos insisten en la dimensión «secreta» o «misteriosa» de la historia que cabe descubrir (Aventures et Dossiers secrets de l'Histoire; Les grands mystères de l'Histoi$r e$ ). Otros se especializan en una región, en general en relación con el patrimonio histórico y cultural (Histoire et Patrimoine; Midi-Pyrénées Patrimoine) o en un periodo histórico (Histoire antique; Moyen Âge; Napoléon Ier), destacando la importancia de los que se interesan en la historia militar, particularmente de la Segunda Guerra Mundial (Batailles. L'bistoire militaire du xxe siècle; Batailles et Blindés; Magazine 39-45). Se podrían añadir títulos relativos a la historia naval, aeronáutica, ferroviaria... O sea que, en la práctica o casi, cada aficionado puede encontrar algún material en torno a su afición.

Volviendo a la diferencia entre las revistas académicas y las revistas de divulgación (obviamente, habría que establecer jerarquías entre unas y otras), digamos rápidamente que hay revistas que solo se consultan, por contener en sus páginas uno o un par de artículos que nos interesan en un momento dado (en relación con la redacción de un trabajo académico, por ejemplo), y otras revistas que se leen en su conjunto como otras revistas de actualidad política o de otra temática. Hay revistas que se venden en kioscos (así como por suscripción individual) y otras que se venden (a veces) en algunas librerías, pero que, por lo esencial, se consultan en bibliotecas (universitarias, por lo general) que adquieren tales revistas por suscripción institucional.

Vincent, M. (2008, 2I de julio). Le magazine L'Histoire a trente ans : auto-célébration, pensée dominante et lois du marché. Documento publicado en la página web de Acrimed (Observatoire des médias) [consultado el I4 de febrero de 20IO] [http://www.acrimed.org/article2936.html]. Ver también NoIRIEL, G. (2005). Les Fils mandits de la République (p. 156). Paris: Fayard. 
En cuanto a las primeras, en la medida en que se dirigen a un público especializado (por áreas de conocimientos), son revistas, por lo tanto, también cada vez más especializadas, bien en totalidad (Annales d'Histoire de la Révolution Française; Guerres Mondiales et Conflits contemporaines, que empezó llamándose Revue de la Deuxième Guerre Mondiale o en parte (con la publicación de dosieres o de números monográficos, en todo caso siempre amenazadas por la competencia de Internet, por lo que se transforman a menudo en revistas electrónicas (caso del Bulletin d'Histoire Contemporaine de l'Espagne), a veces manteniendo al mismo tiempo una versión en papel junto a la versión electrónica4.

La diferencia de presentación entre unas y otras es también evidente y casi definitoria, amén del espacio de venta ya referido: el formato mismo de la revista, su tamaño, el número de páginas, la presencia o no de ilustraciones a todo color, el formato de los artículos..., y naturalmente el contenido de los mismos (erudición especializada, con presencia de notas documentales que precisan las fuentes y la historiografía existente, frente a síntesis más o menos rápida y bien escrita de un episodio histórico, sin notas y [apenas] sin referencias), lo que plantea la cuestión de la historia para el gran público, que no puede (y no quiere) acceder a una revista especializada.

Básicamente, se trata, pues, de la diferencia que puede existir entre un órgano de comunicación en el cual se exponen los resultados de una investigación de alto nivel, en general individual en el sector de las Humanidades, pero también colectiva, como suele ser el caso en Ciencias duras, y la revista de divulgación, que en general no aporta novedades, sino que se contenta con dar a conocer algunos de los resultados alcanzados por los investigadores en función de la demanda social de historia manifestada por el gran público.

\section{El caso de L'Histoire. ¿¿ «a revista de referencia de los apasionados por la historia»?}

La revista L'Histoire, sobre la cual queremos llamar la atención, y que tal vez podría compararse, dentro del mercado español, con la revista catalana L'Avenç, tiene características obvias que la asocian inmediatamente con las revistas de divulgación: el formato alargado, la cubierta en color y títulos que llaman la atención, la presencia de ilustraciones en color, la paginación (Ioo páginas) y, desde luego, la venta en kiosco (con unos importantes resultados de venta ya mencionados, si bien parecen ir a menos, resultado sin duda de la presión hecha por Internet).

Pero, al mismo tiempo, se trata de un instrumento de referencia en el campo historiográfico y que la diferencia de las demás revistas francesas de divulgación histórica (Enquête sur l'Histoire; Historia; Historama; Notre Histoire y los títulos ya citados)'s. ¿Por qué nos referimos a L'Histoire como a un instrumento de referencia? $\mathrm{O}$, al menos, tal como sus responsables lo pregonan en su página web.

4 Rygiel, P. y Noiret, S. (eds.). (2005). Les historiens, leurs revues et Internet (France, Espagne, Italie). Paris: Publibook, $193 \mathrm{pp}$.

5 Pain-Prado, L. (20IO). La Question de l'histoire grand public : étude comparée de deux magazines d'histoire «Historia» et «L'Histoire» 2004-2008. Trabajo de Máster 2 en Historia elaborado bajo la dirección de Bernard Bruneteau. Universidad de Grenoble-II. Documento en línea en la dirección https://dumas. ccsd.cnrs.fr/dumas-00584023/document. 
Un primer elemento de respuesta es que escriben en L'Histoire profesionales de la historia, universitarios (doctores y agrégés 6 , a menudo antiguos alumnos de las Escuelas Normales Superiores, o sea representantes de la élite universitaria francesa) especialistas en sus respectivos campos de trabajo, reconocidos por sus trabajos de investigación histórica. En el primer número de la revista, encontramos así artículos del medievalista Georges Duby o del contemporaneísta René Rémond...

Podemos citar ulteriormente las colaboraciones de Maurice Agulhon, Alain Corbin, Jean Delumeau, François Furet, Jacques Le Goff, Emmanuel Le Roy Ladurie, Claude Mossé, Mona Ozouf, Paul Veyne y un largo etcétera, en el cual encontramos buena parte de los historiadores franceses que suelen colaborar en las revistas académicas de historia. Pero, obviamente, no todos los artículos vienen firmados por tales nombres prestigiosos y la lista de los colaboradores más o menos conocidos es larga.

No obstante, los artículos publicados en L'Histoire responden, por lo tanto, en una versión que podemos calificar de soft, a las exigencias mínimas del trabajo «científico», incluso cuando se trata de una síntesis (notas, bibliografía mínima -titulada «Pour en savoir plus» [Para saber más]-). Por lo tanto, el lector especializado no se encuentra totalmente en otro mundo y el lector aficionado puede acercarse sin mayores problemas a algunas modalidades de presentación de los trabajos especializados. Se trata de trabajos que pueden citarse en un trabajo de investigación «tradicional», lo que difícilmente podría ser el caso de otras revistas de vulgarización, salvo en el caso de un trabajo sobre esas mismas revistas.

La ambición de la revista fue, pues, desde el principio, el facilitar a sus lectores la síntesis de los trabajos llevados a cabo por historiadores profesionales, haciéndose eco de los debates historiográficos y también, y cada vez más, de las cuestiones demandadas por la actualidad política, económica y social, sea nacional o internacional (los atentados terroristas, la pandemia...). Pensar históricamente el presente podría ser un lema de los primeros responsables de los Annales o de Pierre Vilar7.

En sus inicios, el modelo editorial (y el éxito de ventas consiguiente) era la revista de vulgarización científica La Recherche (La Investigación), revista mensual de referencia en el campo científico «duro» (biología, física, química...). Nada extraño que fuera editada al principio por un grupo de prensa (Société d'Éditions Scientifiques, filial de las Ediciones Le Seuil) que también editaba precisamente La Recherche. Además, los «padres» de L'Histoire en 1978 fueron Michel Chodkiewicz, entonces director de La Recherche, y Michel Winock, historiador que era en ese momento director literario en la editorial Le Seuil, conocida por sus publicaciones históricas académicas (Historia de la vida privada...).

La revista se mueve, pues, en el mundo de la empresa privada, característica que no hay que olvidar, pues orienta todos los contenidos de la revista según un criterio de rentabilidad económica. Desde julio de 20I6, la revista es editada por la sociedad

\footnotetext{
6 Recordemos que los agrégés son los titulares de la agrégation, oposición nacional de alto nivel -desde Napoleón I- para reclutar (en principio) a catedráticos de segunda enseñanza (en Letras y en Ciencias). En Derecho y en Medicina, la agrégation es por lo contrario una oposición universitaria. Ver CHERVEL, A. (1993). Histoire de l'agrégation. Contribution à l'histoire de la culture scolaire. Paris: InRP-Éditions Kimé, 289 pp.; GuereñA, J.-L. (2004 [2005]). La Universitat a França: realitats, problemes, transformacions. $R e-$ vista Catalana de Pedagogia, Barcelona, 3, 393-403.

7 Vilar, P. (1995). Pensar històricament. Reflexions i records. Edició preparada i anotada per Rosa Congost. València: Eliseu Climent, Editor (Sèrie «La unitat», n. ${ }^{\circ}$ I56), 339 pp.
} 
Sophia Publications, que pertenece al Grupo Challenges y cuyo único director es el rico industrial y empresario Claude Perdriel (nacido en 1926). Antiguo alumno de la Escuela Politécnica y propietario de varios grupos industriales (bañeras para spas, trituradoras, bombas...), se orientó hacia el mundo de la prensa y de la edición.

En el marco de Sophia Publications, y entre otras revistas que también dirige, Perdriel es el director de publicación de L'Histoire y de la revista de divulgación histórica ya aludida Historia, lo cual no deja de plantear problemas, dadas sus posiciones políticas y su manera activa de darlas a conocer públicamente ${ }^{8}$.

En las últimas elecciones presidenciales francesas, Perdriel apoyó así, de manera abierta, a Emmanuel Macron, entonces el candidato de «En Marche», el actual presidente, en su marcha hacia la conquista del poder. En el marco de su estrategia, L'Histoire publicó una larga entrevista (por el historiador Michel Winock y el periodista Guillaume Malaurie), de cuatro páginas, de E. Macron -el único candidato en disfrutar de esa oportunidad- en las páginas de un número especial de abril de 2017 («La grande querelle: l'histoire de la France»), para que «demuestre hasta qué punto su concepción de la historia participa de su visión de Francia»?.

La directora de la redacción de la revista L'Histoire, Valérie Hannin, es una antigua alumna de ENS de Fontenay (hoy afincada en Lyon junto con la antigua ENS de Saint-Cloud) y agrégée de historia, lo cual la condujo anteriormente a ejercer de profesora en varios institutos de segunda enseñanza antes de pasar a ser elegida para esa función.

En el comité de redacción, se encuentran diversas personalidades más o menos conocidas en el mundo de la historiografía francesa: Pierre Assouline, Jacques Berlioz, Patrick Boucheron, Catherine Brice, Bruno Cabanes, Johann Chapoutot, Joël Cornette, Clément Fabre, Anäs Fléchet, Jean-Noël Jeanneney, Philippe Joutard, Emmanuel Laurentin, Julien Loiseau, Pap Ndiaye, Fabien Paquet, Olivier Postel-Vinay, Yann Potin, Yves Saint-Geours, Maurice Sartre, Claire Sotinel, Pierre-François Souyri, Laurent Theis, Annette y Olivier Wieviorka, y Michel Winock.

Entre los lectores de L'Histoire, podemos distinguir entre el público de los meros «aficionados»-el «gran público» en general, interesado desde luego en la historia y con una relativa familiaridad procedente de los años de aprendizaje escolar, al cual ya nos hemos referido-y, por otra parte, una clientela «cautiva», el público estudiantil, compuesto en particular por los estudiantes que preparan varias oposiciones de alto nivel en donde la Historia es un componente esencial o importante: en la universidad, la agrégation de Historia ya mencionada, y en los institutos (clases de Première Supérieure o Khâgne en argot estudiantil), los temas de Historia en las oposiciones para ingresar en las Escuelas Normales Superiores (París, Lyon, Cachan) o en el Instituto de Ciencias Políticas (comúnmente conocido como «Sciences Po») de París y de otras localidades (Lyon, Lille, Burdeos, Toulouse...).

8 Ebrard, Q. (23 de junio de 2016). Claude Perdriel seul propriétaire de Historia, L'Histoire, Le Magazine Littéraire et La Recherche. Le Monde (documento consultado el Io-XII-2020 en la página http:// www.lemonde.fr/actualite-medias/article/2016/o6/23).

9 Macron, E. (mayo de 2017). «Réconcilier les mémoires», Propos recueillis par Michel Winock et Guillaume Malaurie. L'Histoire, Número fuera de serie, n. ${ }^{\circ} 4$ ( «La grande querelle: l'histoire de la France», texto parcialmente disponible en la página web de la revista [https://www.lhistoire.fr/parution/ hors-s\% $\mathrm{C}_{3} \%$ A 9 rie-4) $]$ y de manera integral en la página de la versión francesa del periódico en línea Huffington Post [actualmente, HuffPost], 22-III-2107 [https://www.huffingtonpost.fr/2017/03/22/interview-demmanuel-macron-avec-lhistoire-reconcilier-les_a_21904543/]. 
En su página web, la revista propone para dichos estudiantes varios «webdossiers» (naturalmente, de pago), reuniendo y presentando algunos artículos ya publicados en torno a un tema de la oposición. Por ejemplo, para el tema de historia contemporánea en la agrégation de 2021, «Le travail en Europe occidentale des années I830 aux années 1930. Mains d'oeuvre artisanales et industrielles, pratiques et question sociale»; para el tema de historia moderna, «Le monde de l'imprimé en Europe occidentale, I470I680», o para el tema de historia antigua, «Religion et pouvoir dans le monde romain». También es el caso para las oposiciones de ingreso en las Escuelas Normales Superiores y en los Institutos de Estudios Políticos.

Pero si esta modalidad de presentación de los artículos publicados es reciente, el conjunto de lo publicado al filo de los años (lo que podríamos calificar de «capital» de la revista) es considerable y se han publicado varios índices temáticos que permiten valorarlo fácilmente.

La revista L'Histoire ha abordado, en efecto, cantidad de temas que han marcado el mundo, desde la antigüedad hasta nuestros días. Cada número de la revista en papel incluye un dosier monográfico constituido por varios artículos relacionados en torno a una temática dada, algunos artículos variados, una crónica de «Actualidad» (exposiciones, coloquios...) y algunas reseñas (en general, de autores relacionados con la revista), así como entrevistas, mapas y cronologías.

En fase con la actualidad política, la revista ha publicado así dosieres acerca de la inmigración o del laicismo, temas de intensa polémica en Francia. También, la publicación otorga gran importancia a la evolución de la sociedad y de las costumbres (y, por lo tanto, de las actitudes frente a la sexualidad), desde la Europa anterior al cristianismo.

Desde febrero de 1998, L'Histoire propone además a sus lectores números especiales (Les Collections de l'Histoire) que ofrecen síntesis sobre una gran variedad de temáticas a partir del «banco» de los artículos publicados y disponibles más unos cuantos inéditos. Cabe señalar así mismo la publicación de atlas históricos dentro de una estrategia de diversificación y complementariedad.

Finalmente, en junio de 2020, vio la luz, con la complicidad de Laurence De Cock, profesora de historia-geografía en un instituto e investigadora en Ciencias de la Educación $^{10}$, una iniciativa interesante, L'Histoire Juniors, publicación en Internet de un dosier de ocho páginas, adaptado de la revista en papel para hacer asequibles a los más jóvenes las principales aportaciones de la investigación histórica sobre temas de su interés.

Citemos los temas de los siete números publicados hasta ahora bajo ese título: «Gare aux sorcières» [«Cuidado con las brujas»], «Lire et écrire au Moyen Âge» [«Leer y escribir en la Edad Media»], «Les Sioux», «Les Gaulois» [«Los Galos»], «I720. la peste à Marseille» [«1720, la peste en Marsella»], «La révolution de I848» [«La revolución de I848»], «Magellan et le tour du monde» [«Magellan y la vuelta al mundo»].

io De Cock, L. y Picard, E. (eds.). (2017). La Fabrique scolaire de l'bistoire. 2. a edición revisada. Marseille: Agone (Collection Passé \& présent), 2I3 pp.; DE Cock, L. (2018). Sur l'enseignement de l'histoire : Débats, programmes et pratiques de la fin du xIxe siècle à nos jours. Paris: Libertalia, 329 pp. 


\section{Algunas breves palabras de conclusión}

La publicación en mayo de 2008, en ocasión de su trigésimo aniversario, de un número especial de la revista, titulado « 30 ans qui ont changé le monde» [«30 años que han cambiado el mundo»], ha suscitado algunas críticas y permite analizar algunas de las razones del éxito de L'Histoire. Matthieu Vincent, en un artículo ya citado, las sintetiza en el título de la manera siguiente: autocelebración, pensamiento dominante y leyes del mercado, lo que podríamos sintetizar por discurso «liberal», anticomunista y antimarxista, lo que se pudo observar en el tratamiento del bicentenario de la Revolución francesa y de algunos de sus actores.

Al leer la revista, y en particular aquel número retrospectivo ya antiguo, parece ser que todo el campo de los estudios históricos gira en Francia en torno a la revista, a sus colaboradores, a las iniciativas y a los debates historiográficos que impulsa, lo que no significa obviamente que el contenido mismo de la revista carezca de valor. Pero merece tenerse en cuenta para evitar una lectura «ingenua» de la revista.

Además, la oferta de historia, en su sentido más amplio, ya no puede reducirse a lo que era en 1978, al salir el primer número de la revista. Aparte de la masa de informaciones que podemos encontrar en Internet, nuevas revistas de divulgación han hecho su aparición, como en 1999 y desde Burdeos, lo que es de notar en un país tan centralista como Francia, La Revue de l'Histoire, lo que le ha permitido alcanzar el n. ${ }^{\circ} 93^{\mathrm{II}}$.

La vulgarización histórica no es nada evidente, sea en un marco oral (conferencia para el «gran público») o escrito (el caso de las revistas que nos ocupa aquí) y conviene analizar tanto la «sed de pasado» del público como las estrategias implementadas en las revistas de divulgación. Como lo señala Olivier Zeller en 2000, algunas revistas de vulgarización buscan la atención de sus lectores por títulos del editor, mitad informativos, mitad provocadores, o al menos atractivos. El artículo, provisto de un título en grandes mayúsculas e ilustrado de la misma manera por el editor, puede correr el riesgo de verse reducido a una suerte de justificación del título impuesto ${ }^{12}$.

Todo ello no limita el interés de la revista L'Histoire como tampoco apreciar su voluntad de partir del presente para abordar históricamente problemas concretos de la actualidad, contestando de tal modo a la demanda social de historia, que también busca respuestas a periodos más antiguos, sea de la época moderna o antigua.

Ver la página web de la revista: http://larevuedelhistoire.com.

12 Zeller, O. L'historien dans la Cité: les pièges de la vulgarisation», en Philippe Fritsch (ed.), Implication et engagement. Hommage à Philippe Lucas, Lyon, Presses universitaires de Lyon, 2000, pp. 26I-270. 